Revue belge de géographie

\title{
Les élections roumaines : le poids du passé
}

Elections in Romania: the weight of the past

\section{Octavian Groza et Catrinel Trofin-Gille}

\section{(2) OpenEdition}

\section{Journals}

Édition électronique

URL : http://journals.openedition.org/belgeo/15382

DOI : 10.4000/belgeo.15382

ISSN : 2294-9135

Éditeur :

National Committee of Geography of Belgium, Société Royale Belge de Géographie

Édition imprimée

Date de publication : 30 juin 2001

Pagination : 123-136

ISSN : 1377-2368

\section{Référence électronique}

Octavian Groza et Catrinel Trofin-Gille, «Les élections roumaines : le poids du passé », Belgeo [En ligne], 1-2 | 2001, mis en ligne le 30 novembre 2001, consulté le 19 avril 2019. URL : http:// journals.openedition.org/belgeo/15382 ; DOI : 10.4000/belgeo.15382

\section{Ce document a été généré automatiquement le 19 avril 2019.}

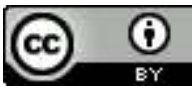

Belgeo est mis à disposition selon les termes de la licence Creative Commons Attribution 4.0 International. 


\section{Les élections roumaines : le poids du passé}

Elections in Romania: the weight of the past

Octavian Groza et Catrinel Trofin-Gille

1 L'an 2000 signifie pour les Roumains les troisièmes élections générales depuis la réinscription de leur pays sur la voie démocratique ${ }^{1}$. Des élections importantes pour le devenir du pays, car l'ascension d'un parti extrémiste (România Mare - La Grande Roumanie), amalgame dangereux de populisme, d'antiparlementarisme, de nationalisme et d'antisémitisme, brouille la bipolarisation entre démocrates et réformateurs d'origine communiste. Des élections surprenantes aussi dans leurs configurations régionales, dont les résultats permettent de reformuler certaines questions portant sur les composantes régionales au sein du territoire national.

2 C'est cette concrétisation régionale des choix de vote des citoyens de Roumanie que nous voudrions examiner, comprendre et interpréter. Comment y lire le poids des vicissitudes du XXe siècle? Dans quelle mesure le vote ethnique, si caractéristique de chaque scrutin, est-il en partie le reflet de la défaillance du politique? Quels sont les signes d'une évolution des comportements, de l'ethnique vers le civique?

\section{L'étouffement communiste}

Dans tout système démocratique le vote est une décision de compromis entre les intérêts individuels à court et moyen terme et les intérêts communautaires à moyen et long terme ; or, actuellement, les objectifs, qu'ils soient du premier ou du deuxième niveau, se modifient extrêmement vite. Les seuls freins à cette dynamique accélérée restent les structures socio-culturelles et économiques incrustées dans l'espace. Ces structures, dans les pays à longue tradition démocratique, représentent le résultat des négociations incessantes entre l'individu et sa communauté et les organisations politicoadministratives régionales ou/et étatiques. 
Dans les pays de l'Europe centre-orientale, ce type de transaction n'a pu se développer que dans la brève période d'entre les deux guerres mondiales et elle a été suivie d'un demi-siècle d'autorité absolue de l'Etat, exercée sur une majorité d'individus assistés, sans aucune propriété et donc sans aucun droit et avec, en plus, leurs intérêts personnels totalement assujettis aux intérêts soi-disant nationaux. La négation systématique des individus et des communautés locales et régionales n'a pas créé pour autant des espaces nationaux homogènes, ni dans la réalité de la construction territoriale, ni dans les représentations individuelles ou collectives concernant cette construction. Les communautés locales ont continué à se définir territorialement par rapport aux repères officieux des anciennes régions historiques. Le caractère officieux de ces constructions territoriales régionales, obnubilées par un centralisme autoritaire de l'Etat, a renforcé l'aliénation et le déracinement des individus. C'était la période où seul avait droit de cité un discours officiel, fondé sur une version de l'identité nationale à forte connotation démagogique.

5 La disparition des régimes politiques autoritaires a soudainement révélé la fragilité des sociétés de l'Europe centre-orientale dont les individus, longtemps privés de réflexion sur eux-mêmes, se comportaient comme des papillons autour des lampadaires des nouveaux discours politiques. La complexité des situations politiques, mise en relief par les renversements électoraux, que ce soit en Pologne, en Hongrie ou en Roumanie, reflète en effet les avatars d'une quête fondamentale d'identité territoriale. Longtemps privés de réelle responsabilité civique, et incertains sur leur conscience d'appartenance à d'autres niveaux territoriaux que celui des régions historiques, les membres de ces sociétés s'avèrent très sensibles aux discours identitaires de type communautaire, fondés soit sur la langue, soit sur l'ethnie, soit sur la confession. Du divorce de velours tchécoslovaque aux nettoyages ethniques de l'ancienne Yougoslavie, toutes les situations sont présentes à l'Est de l'ancienne ligne du rideau de fer.

Nous sommes donc persuadés que toute analyse géographique des manifestations politiques en Europe centre-orientale doit tenir compte à la fois de la fragilité actuelle des comportements individuels et de la puissance des structures territoriales anciennes, comprimées par les régimes communistes, et qui, réapparaissant dans un contexte de mauvaise gestion du territoire, peuvent devenir des graines de catastrophes.

\section{Le vote ethnique ou le politique défaillant}

7 L'analyse des cartes électorales de 1992 et de 1996 montre que l'effet régional le plus puissant est une fonction de la composante ethnique du corps électoral. La dimension ethnique introduit le critère principal de différenciation entre le comportement électoral des habitants du Vieux Royaume, formé à la suite de l'union entre les provinces de Moldavie et de Valachie en 1859 et celui des habitants de la Transylvanie, ancienne province des Habsbourg (1867-1918). Cette empreinte du passé dans l'espace électoral actuel est extrêmement visible, notamment dans les départements qui se rangent sur une diagonale nord-ouest/sud-est, correspondant au territoire annexé par le gouvernement hongrois de Horthy en 1940. Ce territoire constitue le champ principal d'affrontement entre les deux principales formations à base ethnique : l'UDMR (Union Démocratique des Magyars de Roumanie) et le PUNR (Parti de l'Unité Nationale des Roumains). C'est là que les souvenirs des exactions de l'armée horthyste, ravivés par la propagande communiste, sont restés forts dans la mémoire collective. 
Figure 1. Carte des lieux rappelés dans le texte.

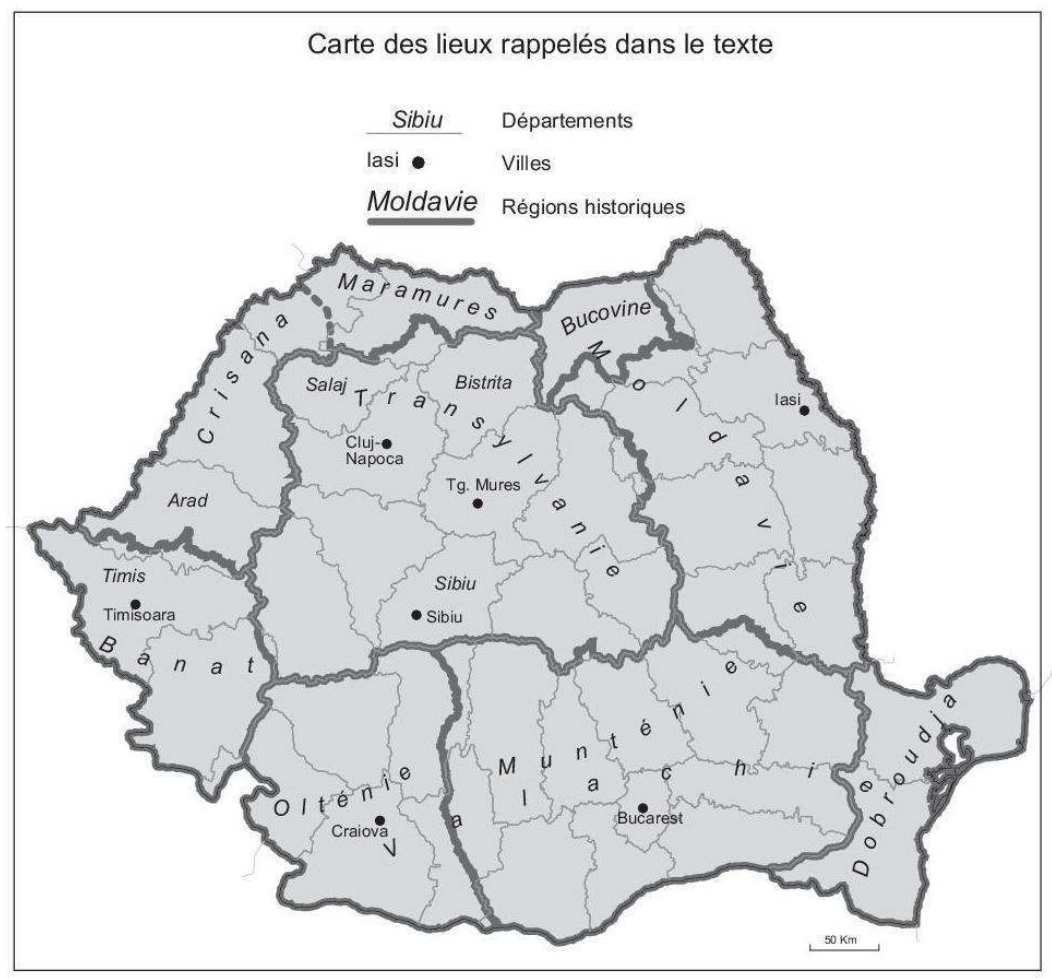

8 La forte composante d'ethnicité sur la scène politique roumaine est aussi la conséquence des évolutions confuses d'après décembre 1989 et du système politique mis en place par l'Assemblée constituante élue en 1990. La Constitution roumaine reconnaît aux associations des minorités nationales un caractère politique en leur garantissant un siège de député, même dans le cas où les résultats obtenus dans les urnes ne le justifieraient pas (figure 2). Cette liberté constitutionnelle, généreuse en intention, peut être un obstacle important sur la voie de la construction d'une attitude civique s'exprimant dans le fait électoral. 
Figure 2. Les élections pour la Chambre des Députés -2000.

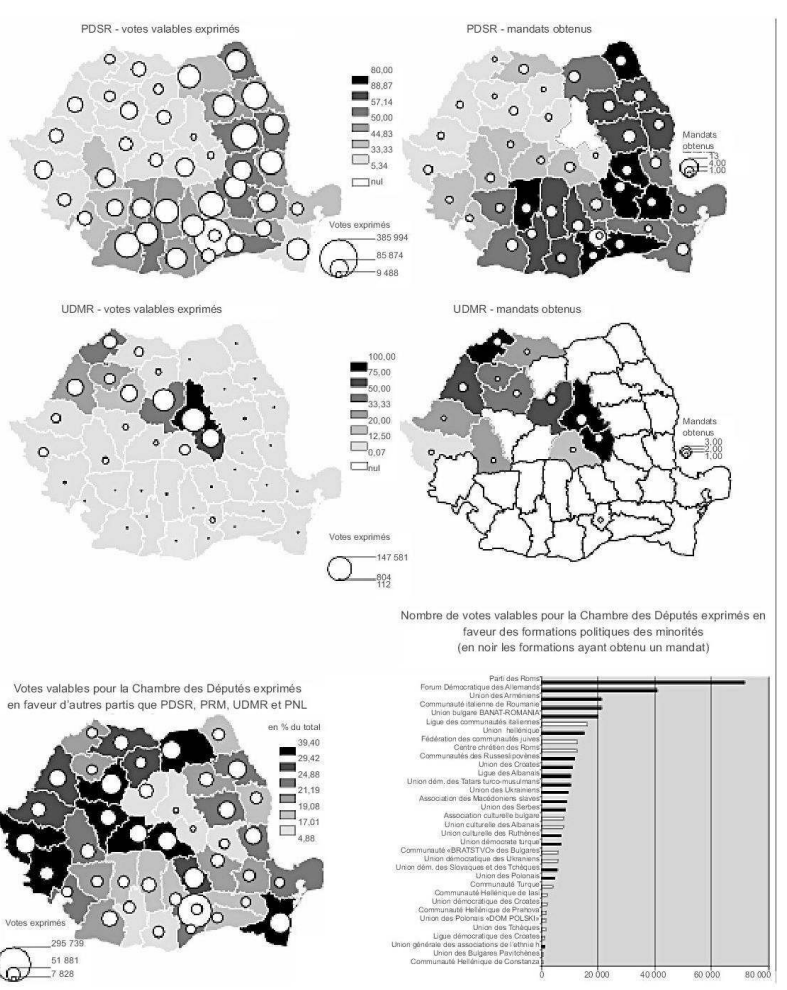

9 La décennie 90 a été marquée par la recomposition d'une bipolarité politique confuse autant que complexe, peu apte à dessiner une direction claire pour les changements nécessaires.

Et pourtant, on pouvait croire en janvier 1990 l'avenir du pays dessiné, comme si la chute du dictateur était suffisante pour ouvrir largement les portes de la démocratie et du consensus national. Toutefois, deux éléments d'effet conjoint opèrent une première scission dans la nouvelle vie politique émergente et mouvementée : le positionnement des acteurs politiques vis-à-vis des héritages idéologiques - l'héritage communiste et l'héritage démocratique de l'entre-deux guerres -, et la géographie de la révolution. Au moment initial du changement de système, le contenu de la bipolarité du monde politique est apparemment peu marqué, mais il s'avèrera lourd de conséquences. Initiée à Timisoara, la révolution éclate quelques jours plus tard à Bucarest et les révolutionnaires des deux villes gardent la même orientation programmatique. Mais bientôt Timisoara, ville martyre, commence à s'opposer à Bucarest, considérant les idéaux de sa révolution trahis par le pouvoir néo-communiste qui prend la direction du pays. Autour des groupes contestataires de Timisoara, qui bénéficient du support des partis "historiques " de l'entre-deux-guerres ${ }^{2}$, commence alors la construction du mythe d'une Transylvanie de la vraie révolution, exigeant la vérité et la démocratie.

Quant aux grands problèmes à résoudre, qui vont rapidement intervenir dans la vie sociale et dans les discours politiques, ils vont augmenter cette bipolarisation initiale et aussi renforcer le sentiment que la Transylvanie reste toujours engagée sur la voie d'une destinée à part. La nécessité de la réforme économique, le respect de la propriété, la désétatisation et la récupération du patrimoine, qu'il soit foncier ou immobilier, et 
surtout l'intégration européenne... font partie des thèmes présents dans tous les programmes politiques, mais bien sûr avec des prises de position totalement divergentes.

De « gauche » comme de «droite », le politique au pouvoir n'a pas su convaincre. Détenu entre 1990 et 1996 par les partis de " gauche ", dont la plupart des membres débarquaient directement du Parti Communiste Roumain et sous la présidence de Ion Iliescu, le pouvoir reste alors marqué par l'idéologie nationaliste et pour cela produit un niveau très général de discours et d'action politique. Au même moment l'opposition « de droite ", composée notamment des partis à support régional et local, met dans ses discours l'accent sur les problèmes concrets des électeurs, tout en critiquant la stratégie nationale choisie par le pouvoir en place. Alors que le pouvoir « de gauche », poussé par son idéologie mais aussi soucieux de préserver la masse de son électorat, conduit une politique de transition lente censée réduire les coûts sociaux, l'opposition milite pour une réforme dure et rapide. Las des résultats ambigus de la réforme douce, l'électorat s'est orienté vers l'opposition, qui a gagné les élections de 1996. Arrivées au pouvoir en 1996, les composantes de la droite n'ont pas été capables d'accomplir ensuite leurs promesses, bien au contraire; d'où la montée de l'extrémisme en 2000.

D'une façon plus spécifique, la mauvaise prise en considération des différences régionales dans les réformes a lourdement grevé l'efficience de l'action politique et a fait perdurer la dimension ethnique dans le politique, tout en ouvrant les portes à l'extrémisme nationaliste. Ainsi, une des premières mesures de la réforme économique a été la décollectivisation des terres, problème majeur pour les régions du Vieux Royaume, à forte densité agricole. Mal appliquée, cette réforme agraire a engendré 10 ans de discussions infinies entre les partis politiques et un appauvrissement grandissant des paysans de la Moldavie et de l'olténie. Dans cette même période, la rétrocession des propriétés immobilières, une attente ardente en Transylvanie, a trop tergiversé. La conséquence en a été la radicalisation des prises de position des diverses forces politiques et culturelles transylvaines et des gestes politiques assez désespérés dans les autres régions historiques se sentant délaissées et mises à l'écart. C'est en ce sens qu'il faut interpréter la création en 1995 à Iasi du Parti des Moldaves, qui compte après les élections de 2000, 4 maires, dont celui de la ville de Iasi (350.000 habitants).

14 L'intégration européenne est une autre pomme de discorde entre les forces politiques. Tandis que pour les partis « de gauche » et leur masse électorale, l'Europe est avant toute chose un arbitre et un juge, voire un professeur autoritaire et distant, sans implication effective, pour «la droite » et pour ses électeurs l'intégration euro-atlantique est un objectif et l'Europe une réalité tangible et désirée. Vu l'ancrage de la gauche dans les départements du Vieux Royaume et celui de la droite en Transylvanie (figure 2), le problème de l'intégration donne alors l'impression d'avoir des dimensions régionales.

15 Comment cette bipolarisation de l'électorat, qui est plus fragile qu'on le croit, se concrétise-t-elle spatialement à travers les résultats des scrutins législatifs et présidentiels de 1992, 1996 et 2000 ?

16 L'analyse des cartes de 1992 et 1996 montre apparemment une préférence plus forte en Transylvanie pour les forces politiques démocrates, groupées autour de la Convention Démocratique de l'ancien président Emil Constantinescu. Le Parti National ChrétienDémocrate des Paysans (PNTCD), qui est le noyau de cette Convention, est un parti né au début du siècle en Transylvanie austro-hongroise. Cette « singularité » transylvaine est à regarder néanmoins avec précautions, car le vote transylvain en faveur d'une réforme accélérée est tout sauf homogène. Ainsi, dans le département de Hunedoara, très peuplé 
et à dominante ouvrière, l'enthousiasme pour une rentabilisation économique, qui pourrait mettre en danger les emplois, est très limité ; c'est d'ailleurs le département transylvain où il n'y a pas eu de majorité en faveur d'Emil Constantinescu en 1996.

Le scrutin de l'an 2000 rend encore plus difficile une lecture régionale différenciant nettement le centre intracarpatique de l'est et du sud de la Roumanie, en particulier si l'on met l'image de «l'attitude démocrate » des Transylvains à l'épreuve des résultats des urnes. L'analyse départementale des majorités relatives des mandats pour la Chambre des députés n'individualise la Transylvanie que par le vote hongrois et par le vote nationaliste roumain (figure 2). Donc, le penchant pour la démocratie des Transylvains semble être mis en doute par le soutien accordé aux extrémistes. En nombre de mandats, le jeu du scrutin à la proportionnelle ${ }^{3}$ fait ressortir la présence du parti extrémiste PRM (Parti « România Mare » - La Grande Roumanie). Le succès de ce parti « România Mare » (27 députés sur les 111 de la Transylvanie) s'appuie certes sur la récupération de l'électorat « ethnique » roumain antérieurement inscrit au PUNR, lequel est actuellement en pleine perte de vitesse, mais surtout il gagne sur d'autres électorats, dans des zones qui n'étaient pas les fiefs traditionnels des nationalistes (Sibiu, Arad, Timis). Au total, le parti « România Mare » détient la majorité des mandats dans le département de Bistrita, il est à égalité avec l'UDMR et le PDSR $^{4}$ en Salaj et à égalité avec le PDSR dans 5 autres départements.

18 La montée en 2000 d'une troisième force politique, qui utilise pleinement la composante extrémiste nationaliste, souligne les défaillances qui perdurent au sein des deux forces qui ont été alternativement au pouvoir : le PDSR, ancien FSN/FDSN ${ }^{5}$ de Ion Iliescu et la Convention Démocratique d'Emil Constantinescu, avec ses alliés, et le caractère confus de la bipolarisation. Les résultats effectifs de l'action de chaque tendance ont très peu satisfait les électeurs, car les élus n'ont mis en œuvre qu'un minimum de leurs programmes, comme si les instruments de l'action échappaient à leur contrôle. Leurs projets sont restés non-achevés, hésitants, contradictoires et n'ont pas donné de sens à cet avenir qui s'ouvrait en 1989. Le vote en novembre 2000 pour le parti extrémiste de « România Mare » de Vadim Tudor rend compte, surtout dans sa dimension nationaliste, d'un mécontentement profond des Roumains face au devenir national. La faible participation au vote renforce l'idée que l'abstention est une contestation du politique à cause des piètres résultats que celui-ci peut présenter, idée clairement confirmée par les sondages d'opinion. Par exemple, le sondage de novembre 2000 de l'institut spécialisé IMAS montrait que la proportion des électeurs ayant confiance dans les structures politiques locales est de $54 \%$, mais les valeurs tombent à $30 \%$ pour la Présidence et à seulement $17 \%$ pour le Parlement.

\section{De l'ethnique vers le civique, via le local}

19 Parallèlement à la défaillance du politique, qui a joué en faveur d'un renforcement apparent de l'attitude ethnique, une autre conscience civique semble émerger dans l'ensemble de la société, comme peut le montrer une analyse fine à l'échelle communale des résultats des élections municipales ${ }^{6}$. Certes, l'image cartographique fournie par celles-ci s'avère très frustrante en première lecture pour le géographe désireux de présenter de «belles » configurations spatiales. La mosaïque qui apparaitt se refuse à toute analyse spatiale, dans le sens classique du terme. A une exception près toujours, et très circonscrite géographiquement : les aires de forte présence des minorités nationales, 
notamment hongroise, où le discours ethnique minoritaire a généré un effet boomerang, en favorisant l'apparition du nationalisme roumain, majoritaire, autochtone, aussitôt institutionnalisé en parti (1990) à travers des forces politiques extrémistes. Hors ces legs historiques localisés, une simple analyse des cartes des élections municipales de 1992 et de 1996 montre que le nationalisme électoral est très peu présent dans le reste du territoire, ce qui rend fragile l'étiquette de "nationalistes » si souvent et si facilement collée en bloc aux Roumains. Quant à la progression entre 1992 et 1996 du parti "România Mare ", nous avons déjà dit qu'elle relève d'abord d'une exaspération globale contre tous les autres partis, tous également " inefficaces ", et qu'elle montre la force que prend dans de telles circonstances un discours extrémiste fondé sur une critique caricaturale des réalités politiques et économiques du pays, avec une copieuse infusion de xénophobie et de racisme.

L'analyse électorale de la Transylvanie, vue à travers les scrutins municipaux, ne peut guère être réduite à l'opposition schématique entre Roumains et Magyars, ni à l'opposition simple entre un espace qui aurait embrassé le civisme occidental, et le Vieux Royaume, pour lequel la démocratie serait une sorte de «prêt-à-porter » d'importation occidentale. Même si certains continuent d'affirmer que le taux supérieur de présence aux urnes enregistré en Transylvanie est dû principalement à des comportements modernes, de type centre-européen, le tableau des corrélations entre les variables électorales et les variables ethno-confessionnelles montre autre chose en arrière-plan. 
Tableau 1. Le comportement électoral en Roumanie : entre civisme et ethnicité (tableau des corrélations entre les variables électorales des élections locales de 1992 et 1996* et les variables ethno-confessionnelles, à l'échelle des 2949 communes urbaines et rurales).

\begin{tabular}{|c|c|c|c|c|c|c|c|c|c|c|c|c|c|c|c|c|}
\hline & $\mathrm{T} \times 92$ & GA92 & DR92 & NR92 & UD92 & $T \times 96$ & GAS6 & DR96 & NR96 & UD96 & ORTH & CATH & REPR & ROUM & HONG & TZIG \\
\hline TX92 & 1,00 & & & & & & & & & & & & & & & \\
\hline GA92 & $-0,18$ & 1,00 & & & & & & & & & & & & & & \\
\hline DR92 & $-0,16$ & $-0,26$ & 1,00 & & & & & & & & & & & & & \\
\hline NR92 & 0,12 & $-0,38$ & $-0,17$ & 1,00 & & & & & & & & & & & & \\
\hline UD92 & 0,31 & $-0,60$ & $-0,35$ & 0,07 & 1,00 & & & & & & & & & & & \\
\hline TX96 & 1,00 & $-0,18$ & $-0,16$ & 0,12 & 0,31 & 1,00 & & & & & & & & & & \\
\hline GA96 & $-0,04$ & 0,44 & 0,04 & $-0,15$ & $-0,46$ & $-0,04$ & 1,00 & & & & & & & & & \\
\hline DR96 & $-0,14$ & 0,04 & 0,33 & $-0,03$ & $-0,28$ & $-0,14$ & $-0,09$ & 1,00 & & & & & & & & \\
\hline NR96 & 0,03 & $-0,09$ & $-0,09$ & 0,46 & $-0,07$ & 0,03 & $-0,27$ & $-0,08$ & 1,00 & & & & & & & \\
\hline UD96 & 0,31 & $-0,59$ & $-0,32$ & 0,09 & 0,95 & 0,31 & $-0,45$ & $-0,27$ & $-0,07$ & 1,00 & & & & & & \\
\hline ORTH & $-0,31$ & 0,60 & 0,21 & $-0,11$ & $-0,84$ & $-0,31$ & 0,47 & 0,21 & 0,02 & $-0,84$ & 1,00 & & & & & \\
\hline CATH & 0,16 & $-0,39$ & $-0,10$ & 0,02 & 0,54 & 0,16 & $-0,31$ & $-0,12$ & $-0,05$ & 0,52 & $-0,74$ & 1,00 & & & & \\
\hline REPR & 0,29 & $-0,51$ & $-0,27$ & 0,13 & 0,78 & 0,28 & $-0,38$ & $-0,22$ & $-0,01$ & 0,79 & $-0,71$ & 0,13 & 1,00 & & & \\
\hline ROUM & $-0,32$ & 0,61 & 0,24 & $-0,09$ & $-0,88$ & $-0,32$ & 0,47 & 0,24 & 0,07 & $-0,87$ & 0,88 & $-0,58$ & $-0,73$ & 1,00 & & \\
\hline HONG & 0,31 & $-0,61$ & $-0,31$ & 0,08 & 0,97 & 0,31 & $-0,47$ & $-0,27$ & $-0,07$ & 0,96 & $-0,88$ & 0,58 & 0,79 & $-0,91$ & 1,00 & \\
\hline TZIG & 0,15 & $-0,16$ & $-0,01$ & 0,15 & 0,13 & 0,15 & $-0,10$ & 0,01 & 0,08 & 0,12 & $-0,13$ & 0,01 & 0,16 & $-0,32$ & 0,12 & 1,00 \\
\hline AUTR & 0,03 & $-0,07$ & 0,13 & $-0,03$ & $-0,02$ & 0,03 & $-0,06$ & $-0,01$ & $-0,05$ & $-0,03$ & $-0,19$ & 0,15 & $-0,04$ & $-0,34$ & $-0,02$ & 0,03 \\
\hline
\end{tabular}

* A l'heure de la rédaction de cet article, les données à l'échelle communale des élections de novembre 2000 ne sont pas encore disponibles.

TX $(92,96)$ = taux de participation ; GA(92,96) = pourcentage des votes obtenus par les partis « de gauche »; $\mathrm{DR}(92,96)$ = pourcentage des votes obtenus par les partis « de droite »; $N R(92,96)=$ pourcentage des votes obtenus par les partis nationalistes roumains; UD $(92,96)=$ pourcentage des votes obtenus par le UDMR, parti ethnique des magyars; ORTH = pourcentage des orthodoxes dans la population communale $; \mathrm{CATH}=$ pourcentage des catholiques (romains et gréco-catholiques) dans la population communale $;$ REPR = pourcentage des réformés et des protestants dans la population communale ; ROUM = pourcentage des Roumains dans la population communale ; $\mathrm{HONG}=$ pourcentage des Hongrois dans la population communale ; TZIG = pourcentage des Tziganes dans la population communale; AUTR = pourcentage des autres minorités dans la population communale.

Le taux supérieur de participation est directement lié à la minorité hongroise, qui va en bloc aux urnes (corrélation de 0,97 en 1992 et 0,96 en 1996). Il s'agit là d'un comportement minoritaire classique. Forte de l'existence de sa propre formation garantie par la Constitution (en fait, une union qui regroupe plusieurs tendances), la minorité la soutient et ne semble guère avoir en vue d'autres devoirs civiques pour ce qui est de l'avenir du pays entier, puisque les corrélations avec les partis de gauche ou de droite restent négatives aux deux dates. La même chose est imputable aux Roumains de Transylvanie, travaillés par l'action des partis nationalistes, pour lesquels le vote est devenu une affaire ethnique plutôt que civique. Qui plus est, aux élections législatives et présidentielles de 2000 , le taux de participation n'est plus un signe du « civisme transylvain », puisqu'on constate une plus grande participation dans les départements du Vieux Royaume (figure 3). Ce taux d'absentéisme de 2000, plus grand en Transylvanie que dans les autres régions roumaines, traduit en fait le dilemme moral d'un grand nombre d'électeurs et leur refus profond envers les deux candidats. Etait-ce pour autant la meilleure solution que de rester en dehors de la joute électorale, alors que seule une participation massive des électeurs démocrates aurait pu cantonner la montée extrémiste? 
Figure 3. La présence aux urnes - 1996 et 2000.

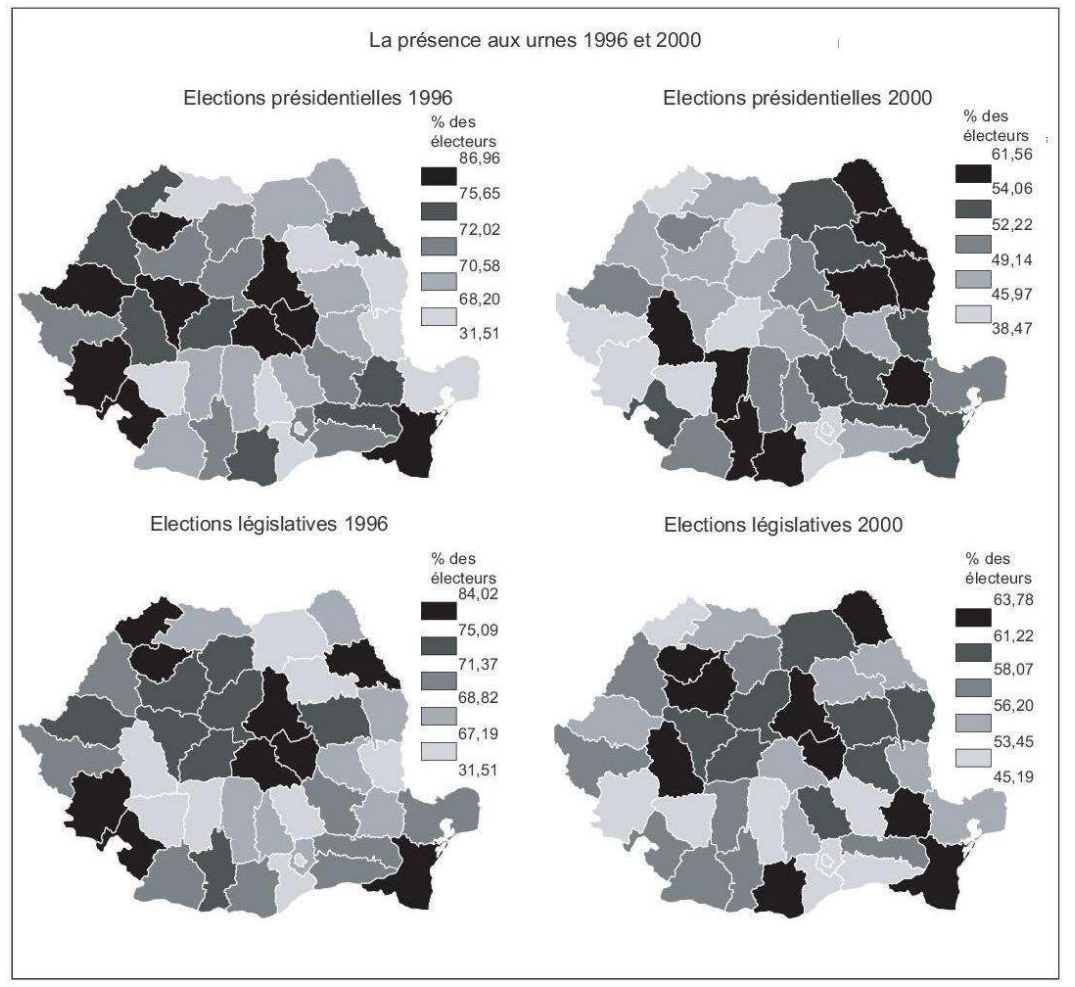

Source : Bureau électoral central

Les élections municipales montrent davantage le besoin d'ancrer la chose politique dans des horizons locaux, ce qui est à la fois très banal et précisément l'objet même des scrutins à ce niveau, dans tous les pays. Toutefois ici, la part prise par des petits partis sans audience nationale et par des personnes localement appréciées mais sans appartenance ( 88 formations politiques présentes en 2000) souligne une fois encore le peu d'emprise et d'impact des programmes des grands partis, incapables d'adapter leurs discours généraux à l'échelon d'une consultation municipale. La mosaïque spatiale décrit un choix de vote structuré par les intérêts locaux et individuels, dont la réalisation est à la fois la condition fondamentale pour construire une identité territoriale solide et sa garantie de durée. En 1992, 31 \% des votes pour les conseillers municipaux revenaient aux petits partis ; en 1996, c'était $44 \%$, taux qui s'est maintenu en 2000 . Au total en 2000 , on a $35 \%$ des maires, $36 \%$ des mandats de conseillers municipaux et $34 \%$ des mandats de conseillers départementaux qui relèvent de cette catégorie " autres partis et formations » (tableau 2). 
Tableau 2. La part des « autres » : les résultats des élections locales de juin 2000.

\begin{tabular}{|c|c|c|c|c|c|}
\hline & Maires & $\begin{array}{l}\text { Conseils } \\
\text { municipaux }\end{array}$ & $\begin{array}{l}\text { Conseils } \\
\text { municipaux }\end{array}$ & $\begin{array}{l}\text { Conseils } \\
\text { départe- } \\
\text { mentaux }\end{array}$ & $\begin{array}{l}\text { Conseils } \\
\text { départe- } \\
\text { mentaux }\end{array}$ \\
\hline & $\begin{array}{l}\% \text { des } \\
\text { votes }\end{array}$ & $\begin{array}{l}\% \text { des } \\
\text { mandats }\end{array}$ & $\begin{array}{l}\% \text { des } \\
\text { votes }\end{array}$ & $\begin{array}{l}\% \text { des } \\
\text { mandats }\end{array}$ & $\begin{array}{l}\% \text { des } \\
\text { votes }\end{array}$ \\
\hline $\begin{array}{l}\text { Parti de la Démocratie } \\
\text { Sociale de Roumanie }\end{array}$ & 36,74 & 28,65 & 25,78 & 28,87 & 27,44 \\
\hline Parti Démocratique & 12,89 & 13,75 & 10,40 & 11,93 & 9,91 \\
\hline Parti National Libéral & 8,40 & 10,02 & 7,80 & 9,31 & 6,96 \\
\hline $\begin{array}{l}\text { Union Démocratique des } \\
\text { Magyars de Roumanie }\end{array}$ & 4,29 & 6,17 & 5,50 & 7,86 & 6,27 \\
\hline Parti «România Mare» & 2,80 & 5,30 & 6,19 & 8,32 & 6,62 \\
\hline Autres partis et formations & 34,88 & 36,11 & 44,33 & 33,71 & 42,80 \\
\hline
\end{tabular}

Source : Bureau électoral central programme d'envergure nationale sont en corrélation négative aux deux dates, ce qui exprime la préférence hongroise pour une vision politique directement hongroise. Cependant, entre 1992 et 1996 la valeur des coefficients diminue sensiblement (de -0,612 à - 0,312 avec les partis de gauche -PDSR, PD, PDAR - et de -0,472 à -0,270 avec les partis de droite -PNL, $\left.\mathrm{CDR}^{8}\right)$. En d'autres termes, une minorité croissante d'électeurs magyars exprime sa lassitude vis-à-vis des luttes intestines de son parti, l'UDMR, vis-à-vis de son discours trop ethnique et idéologique qui ne répond plus aux aspirations personnelles des individus, et manifeste l'amorce d'une prise en considération différente des problèmes politiques. La même chose est valable si l'on choisit l'entrée par les confessions: les catholiques, les réformés, les protestants incluent, aux côtés des Magyars, des Roumains, des Ceangaï (catholiques de Moldavie, d'origine controversée), des Allemands, des Tchèques, des Slovaques, des Italiens, des Polonais et bien d'autres (tableau 1). Qu'observe-t-on en 2000 ? L'élection d'un maire d'ethnie allemande à Sibiu, certes jadis fondation saxonne, mais maintenant à majorité roumaine (moins de $1 \% \mathrm{~d}$ 'Allemands) ou bien de maires d'ethnie roumaine dans des villes (Târgu Mures) ou des communes à majorité hongroise. Il y a là autant de pas vers une attitude civique de l'acte électoral.

de cette même attitude, mais dans le registre des élections présidentielles de 2000 le cas de la mise en échec de la montée extrémiste représentée par le candidat Corneliu Vadim Tudor du Parti « România Mare » (figure 4). Malgré les doutes qui planaient sur son adversaire, Ion Iliescu, au vu à la fois de son passé de nomenklaturiste communiste et de son action comme président entre 1990 et 1996, c'est cependant celui-ci qui a été élu. Le candidat du PRM a été battu grâce aux efforts communs de tendances politiques jusqu'alors opposées; l'attitude exceptionnelle de la communauté hongroise, qui avait 
voté systématiquement contre Iliescu aux élections antérieures, témoigne bien sûr de la défense de ses propres intérêts, mais aussi de la prise en compte d'un intérêt national commun.

Figure 4. Les élections présidentielles - second tour.

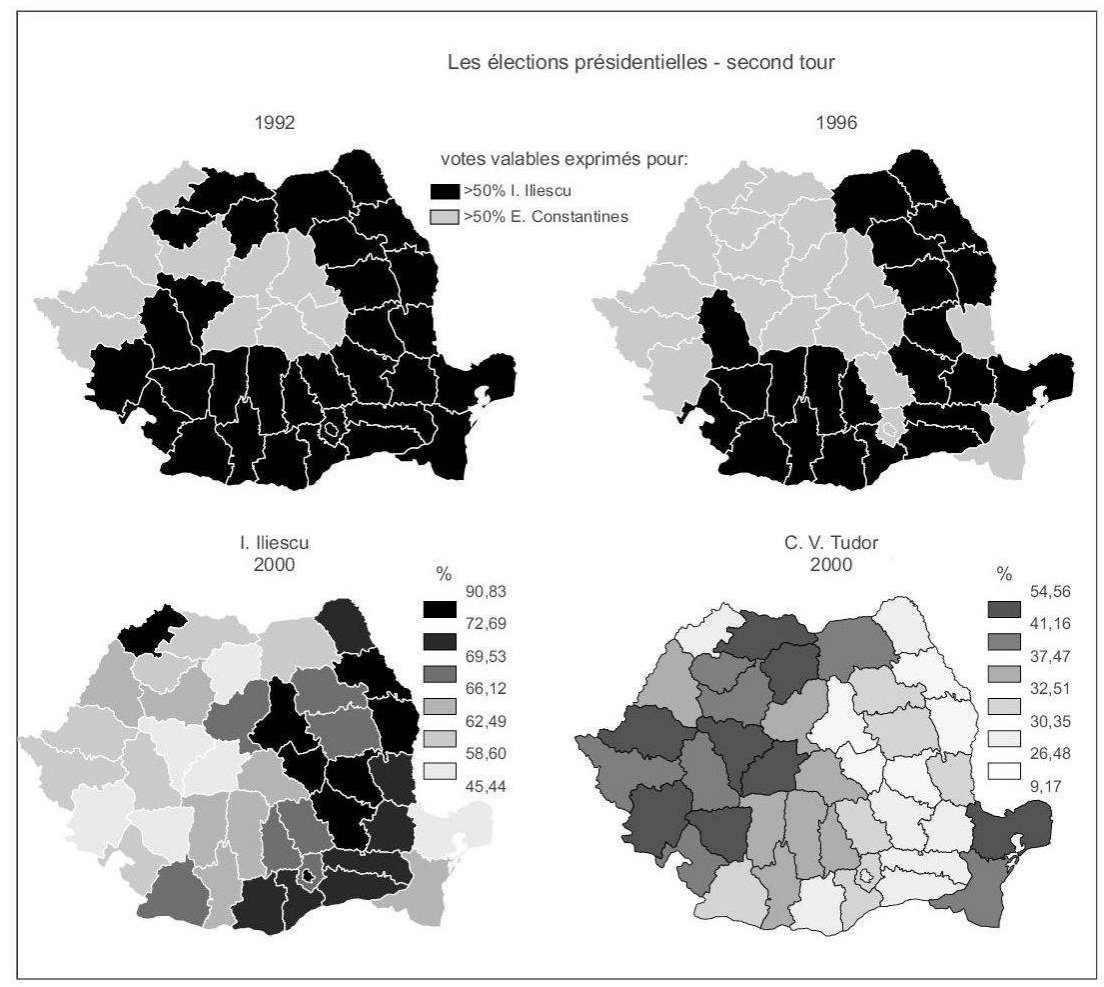

Cette évolution des comportements électoraux de l'ethnique vers le civique est donc manifeste, quoique encore modeste. Elle reste potentiellement tributaire aussi d'un autre impact, celle de l'ethnie rom. Jusqu'à ce jour les membres de cette communauté, évaluée à un demi-million de personnes par le recensement général de la population de 1992, n'ont donné que 80.000 voix aux formations censées les représenter (figure 2); mais qu'adviendrait-t-il s'ils se construisaient une identité politique autonome forte ? Saurontils trouver dès le début de leur intégration communautaire à l'échelle nationale la dimension civique de l'acte public, ou bien les frustrations accumulées les conduiront-ils vers des comportements électoraux sous l'emprise de leur ethnicité?

\section{Conclusions}

Après 10 ans de pratiques électorales démocratiques, la presque majorité des électeurs de Roumanie en était arrivée... à détester le politique, et à n'être guère plus de $50 \%$ à se présenter aux urnes, que ce soit pour des consultations locales ou nationales ${ }^{9}$. De ce bref parcours, il ressort à la fois le poids du passé, un certain refuge vers l'ethnique devant la défaillance du politique, mais aussi l'amorce d'un changement d'attitude vers une conscience civique. Reste une question de fond, qui touche à la structure même du système électoral, avec son système de listes à représentation proportionnelle, tant pour les conseils locaux, les conseils des départements, les députés que les sénateurs, trop favorable à la fragmentation et qui maintient la longévité politique de multiples 
formations. Ce grand nombre de partis participant aux élections a montré d'une manière constante que la scène politique roumaine n'est pas encore complètement structurée. Si ce morcellement politique était compréhensible juste après 1989, il pose aujourd'hui des questions sur la capacité du politique à fédérer des intérêts individuels au sein d'une communauté. Un premier pas a été fait en 2000, avec l'augmentation du seuil d'accessibilité au Parlement de 3 à $5 \%$ des voix, ce qui rééquilibre un peu cette situation de surreprésentation des faibles tendances politiques existantes dans la société et ne peut que réduire l'éparpillement. Le nouveau pouvoir en place depuis décembre 2000 a promis de modifier profondément le système électoral ; de plus, il manifeste une grande fébrilité pour sortir les réformes de l'enlisement et pour changer radicalement les structures. Le fait que presque le quart d'un électorat - qui a déjà une participation modeste aux urnes éparpille ses voix sur de petits partis ou alliances et l'avertissement des électeurs exaspérés auraient-ils déjà un effet sur la classe politique, devenue consciente que, sans un encadrement clair, le chemin vers une attitude civique des électeurs pourrait s'avérer dangereusement long?

Comment expliquer les résultats différents selon les régions et donc les tensions qui en résultent ? La réponse réside dans l'opposition sourde entre le mythe national construit à partir de 1848 et exacerbé après 1920 et les réalités régionales et locales, dont les différences sont restées bien marquées, malgré le passage brutal du rouleau-compresseur communiste. Dans la foulée des mouvements révolutionnaires du XIXe siècle et dans le contexte de la naissance des consciences nationales, la Roumanie n'a pas été une exception en jouant sur la corde ethnique dans la symphonie des nations naissantes. Cette attitude politique aujourd'hui à juste titre bannie impliquait en effet la construction de toute une mythologie du même sang, de la même langue et de la même foi. En bref, cela menait à l'institutionnalisation du patriotisme ethnique. Le processus a été exacerbé une fois la Première Guerre finie, après le découpage des Etats de l'Europe centre-orientale selon le principe des nationalités dominantes. Si en Occident le patriotisme ethnique a évolué vers le patriotisme civique, les régimes autoritaires de la guerre froide ont renforcé les anciennes tendances, d'où l'expansion des nationalismes de toutes sortes, et l'imposition des identités nationales à défaut des identités régionales et locales. Malgré la pression idéologique, les identités infranationales ont perduré grâce aux diverses inerties territoriales, mais la plupart sont sorties déformées de l'étau communiste. Après l'implosion du camp communiste, les sociétés de l'Est, comme la Roumanie d'aujourd'hui, essayent désespérément de retrouver la voie perdue, la voie normale du civisme. Cet article montre que parfois le chemin est long et que l'espoir doit rester vif: les dynamiques en marche semblent avoir retrouvé la bonne direction.

\section{NOTES}

1. Le calendrier des élections après 1989:

- 1990: premières élections libres, institutions provisoires, élection d'un Parlement qui joue le rôle d'Assemblée constituante; 
- février 1992: élections locales; septembre 1992: élections législatives et présidentielles; - juin 1996: élections locales; novembre 1996: élections législatives et présidentielles; - juin 2000: élections locales; novembre 2000: élections législatives et présidentielles.

2. Il s'agit du PNL, le Parti National Libéral, et du PNTCD, le Parti National ChrétienDémocrate des Paysans.

3. Le système électoral roumain est fondé sur un scrutin de liste à représentation proportionnelle. La répartition des votes combine plusieurs méthodes classiques et comporte deux étapes. Il y a d'abord la répartition au niveau du département-circonscription en fonction d'un coefficient électoral propre. Ensuite, les votes restants sont additionnés par parti au niveau national. A l'aide du système d'Hondt, utilisé en Belgique, on calcule les mandats qui restent à distribuer à chaque parti. La distribution par circonscription des mandats restants, ainsi calculés, est effectuée par des méthodes statistiques complexes. Dans le cas où des mandats n'ont pas pu être distribués par ces méthodes, ce qui est exceptionnel, interviennent les négociations et les accords entre les partis concernés.

4. PDSR - Parti de la Démocratie Sociale de Roumanie, le parti gagnant des élections de 2000.

5. FSN/FDSN - Front (Démocratique) du Salut National, le principal parti en 1990-1991.

6. REY V. (2001), «Choix de vote et lieux de vie: analyse géographique des résultats électoraux des villes et des villages de Roumanie (1992-1996)», Belgeo, 1-2, pp. 137-146.

7. PD - Parti Démocrate de l'ancien Premier ministre Petre Roman; PDAR - Parti Démocrate Agrarien de Roumanie.

8. CDR - Convention Démocrate de Roumanie, alliance de partis autour du PNTCD, détruite après les mauvais résultats des élections de 2000.

9. 56,52\% aux élections législatives de 2000 .

\section{RÉSUMÉS}

Depuis 1989, la Roumanie connaît les avatars de la redécouverte des dimensions locales et régionales de la construction territoriale. Le phénomène est très compliqué à cause de la complexité du territoire roumain, caractérisé par l'existence de tensions profondes dues à la jeunesse et à la rapidité de la construction de l'Etat moderne roumain. Cet article explore, à travers une analyse géographique des élections, la diminution des tensions ethniques et la lente émergence d'un comportement civique des habitants de la Roumanie. Le processus est caractérisé par un début d'affirmation des structures locales et régionales comme réaction aux tendances centralisatrices dues à l'héritage communiste.

Since 1989 Romania has been experiencing the avatars of the rediscovery of the local and regional dimensions of the territorial building. The phenomenon is very complicated because Romania is a complex territory characterized by deep tensions determined by the quick and recent construction of the modern Romanian State. This paper explores, through a geographical analysis of the elections, the decrease of the ethnical tensions and demonstrates the slow emergence of a civic behaviour on the part of Romanian citizens. The process is characterized by 
a slow affirmation of local and regional structures as a reaction to centralizing trends inherited from communism.

INDEX

Mots-clés : géographie électorale, vote ethnique, comportement civique, Roumanie Keywords : electoral geography, ethnic behaviour, civic behavior, Romania

\section{AUTEURS}

\section{OCTAVIAN GROZA}

Département de géographie, Université « Alexandru Ioan Cuza » Iasi, Roumanie, ogroza@gemma.geo.uaic.ro

\section{CATRINEL TROFIN-GILLE}

Géophile, ENS -LSH Lyon, France 